\title{
Populations of galaxy groups in clusters from wide-field multicolor imaging
}

\author{
I. H. Li ${ }^{1}$, H. K. C. Yee ${ }^{1}$, E. Ellingson ${ }^{2}$ and G. Laurent ${ }^{2}$ \\ ${ }^{1}$ Department of Astronomy and Astrophysics, University of Toronto, 60 St. George Street, \\ Toronto, ON, M5S 3H8, Canada email: tornado@astro.utoronto.ca, hyee@astro.utoronto.ca \\ ${ }^{2}$ Center for Astrophysics and Space Astronomy, CB389, University of Colorado, Boulder, \\ CO 80309, USA email: elling@casa.colorado.edu, Glenn.Laurent@Colorado.EDU
}

\begin{abstract}
We present the preliminary results of $B V R I$ imaging of six clusters chosen from a follow-up project of the $C N O C$ cluster survey. In an attempt to ascertain the evolution of galaxies in the cluster environment, we focus on the infalling galaxy groups, especially on their characteristics reflected by their color properties. To investigate the role of cluster environment in the evolution of galaxy groups, the color properties of galaxy groups are explored as a function of cluster radius and the local density of cluster galaxies. Both the radius and the local density affect the properties of groups, but evidence suggests that density may be more important in the relationship between cluster environment and galaxy characteristics.
\end{abstract}

\section{Introduction}

Clusters grow via the accretion of the field galaxies or galaxy groups. A general picture of clusters is that star-forming blue galaxies are in the suburbs while the central regions are occupied by the red passive galaxies. The infalling field galaxies and groups evolve and change their stellar population under the influence of the cluster environment.

This work focuses on the infalling groups rather than individual galaxies. The gravitational potential well possessed by groups could affect the evolution of grouped galaxies. To change the distribution of infalling galaxy populations to what we observe in clusters, the infalling groups must evolve to turn off their star forming activities while or before being accereted by the clusters. This change can be reflected in the color properties. This report presents the preliminary results of $B V R I$ four-color imaging of six galaxy clusters at $0.17 \lesssim z \lesssim 0.38$. We adopt $\Omega_{m}=0.3, \Omega_{\Lambda}=0.7$, and $H_{0}=75 \mathrm{~km} / \mathrm{s} / \mathrm{Mpc}$.

\section{Data and analysis}

Sample and data: We have observed six clusters in $B V R I$ from $C N O C$ cluster survey (Yee et al. 1996) using CFH12k mosaic camera with a $28^{\prime} \times 42^{\prime}$ field of view. We formed photometric-redshift samples of galaxies for each cluster with an absolute magnitude limit of $M_{R}=-19.0$.

Photometric redshift method: To select the cluster galaxies, we use the empirical four-color fitting photometric redshift method based on that developed by Connolly et al. (1995). The CNOC2 field galaxy survey (Yee et al. 2000) patch 0920+37 is used as the training set. Both color and magnitude are used in the fitting. Different photometric redshift cutoffs based on the dispersion in $z_{\text {spec }}-z_{\text {phot }}$ as a function of galaxy color are used to select galaxies in the cluster redshift space. The $1 \sigma$ redshift cutoffs range from 0.03 to 0.05 . 

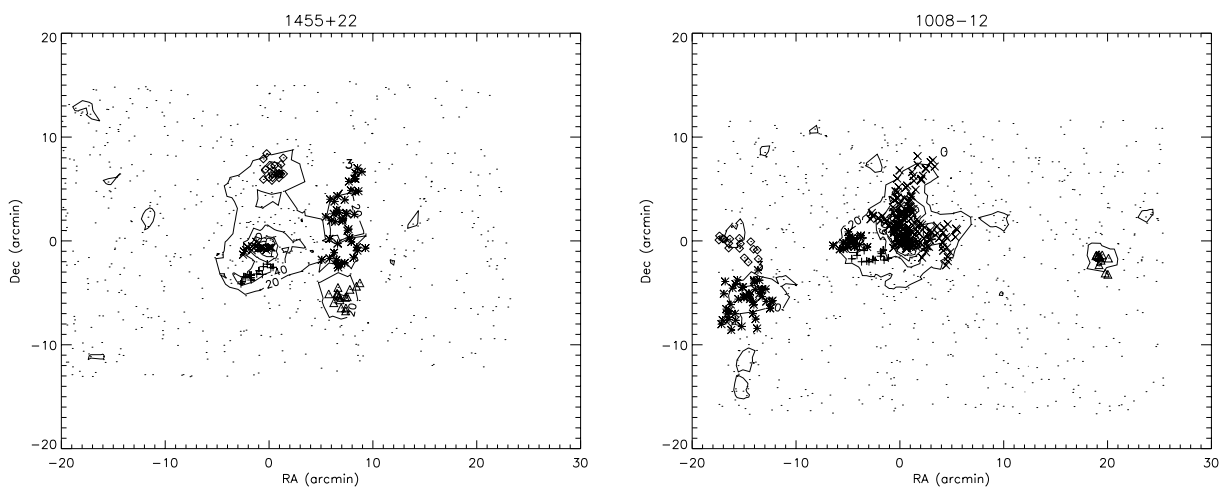

Figure 1. Map of (a) MS1455+22 (b) MS1008-12. Contours are based on the local galaxy number density without background and foreground subtraction. Different groups, as found by a friends-of-friends algorithm, are marked by different symbols.

Background and foreground determination: The background and foreground contamination are determined by using the same CNOC2 patch (with an effective area of $657 \operatorname{arcmin}^{2}$ ) with the same magnitude cutoffs as individual clusters.

Group finding: To see the local environmental effect, the local surface galaxy number density $(\Sigma)$ is calculated based on the distance to the $10^{\text {th }}$ nearest neighbor, $r_{10}$. A friends-of-friends algorithm is used to find galaxy groups. To differentiate galaxy groups in different cluster global density regions, we use a dynamic linking length. Examples of cluster maps and groups are shown in Fig. 1. The dynamic linking length, a function of cluster galaxy density and cluster-centric radius, can uncover groups even in the high density regions close to the cluster cores.

\section{Discussion}

Cluster environmental effects: As an example, we plotted $B-R$ color as function of cluster-centric radius (Fig. 2a) and local galaxy density (Fig. 2b) for MS1455+22. The color gradient becomes nearly constant after passing $R_{200}$, suggesting that the color properties strongly correlate with cluster-centric radius within the virial radius. We also see a drop in the color trend at $1.2 \leqslant \log \Sigma \leqslant 2.0$ galaxies $/ \mathrm{Mpc}^{2}$. This color transition may indicate a threshold effect in transforming galaxy properties (Kodama et al. 2001; Gomez et al. 2003).

Blue galaxy fraction: Figure 3 shows the cluster blue fractions (with different magnitude cutoffs) as a function of cluster-centric radius (normalized by $R_{200}$ ). The blue fractions calculated using fainter magnitude cutoffs are higher, reflecting that faint galaxies are usually blue. The blue fractions at the virial radius are similar for different clusters, regardless of cluster richness. The blue fraction also tends to increase with decreasing local density (Fig. 4) for galaxies in both groups and clusters, indicating that local environment is more important than the average cluster environment. In the same density region, high- $z$ clusters have larger fractions of blue galaxies. Similarly, groups in high- $z$ clusters tend to contain more blue galaxies, suggesting that the Butcher-Oemler effect may also be seen in groups. 

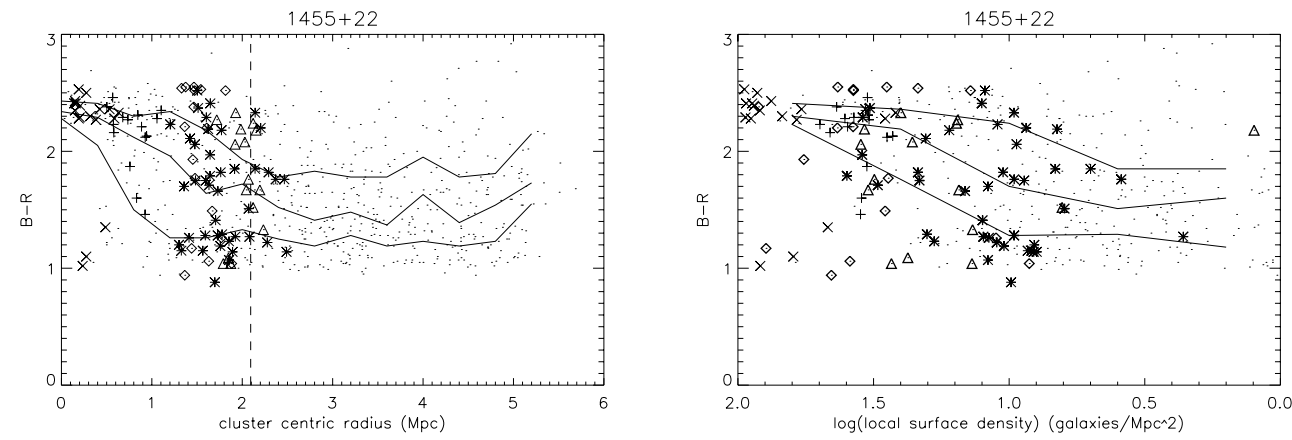

Figure 2. $B-R$ color as a function of $(a)$ cluster-centric radius (b) local galaxy density for MS1455+22. Overlayed are 75, 50, and 25 percentile of galaxy color. Galaxies in groups are marked by different symbols. The vertical dashed line in (a) indicates $R_{200}$.
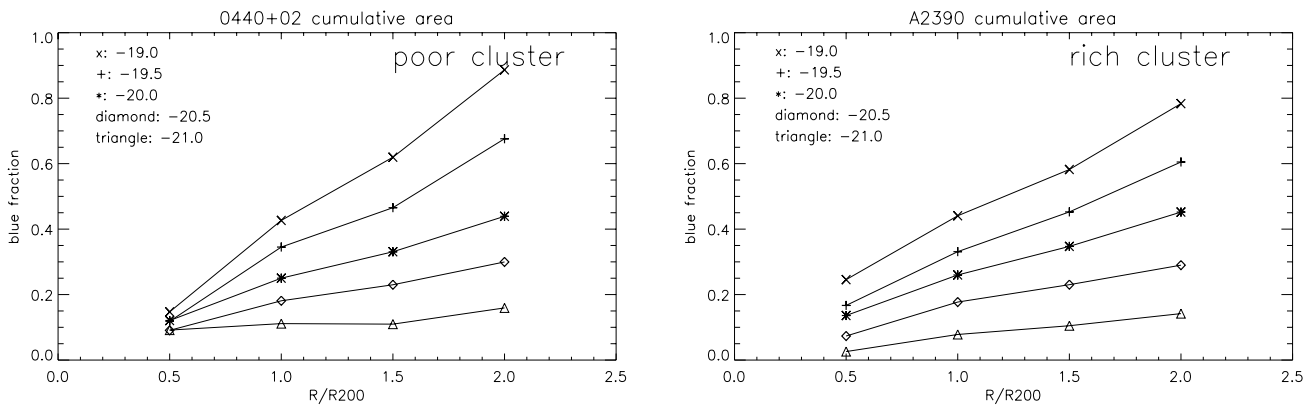

Figure 3. Blue fraction as a function of cluster-centric radius (a) MS0440+02 (b) Abell 2390. Several magnitude cutoffs are used as indicated in the upper-left in the plots. The blue fractions are similiar at $1 R_{200}$ regardless of cluster richness.
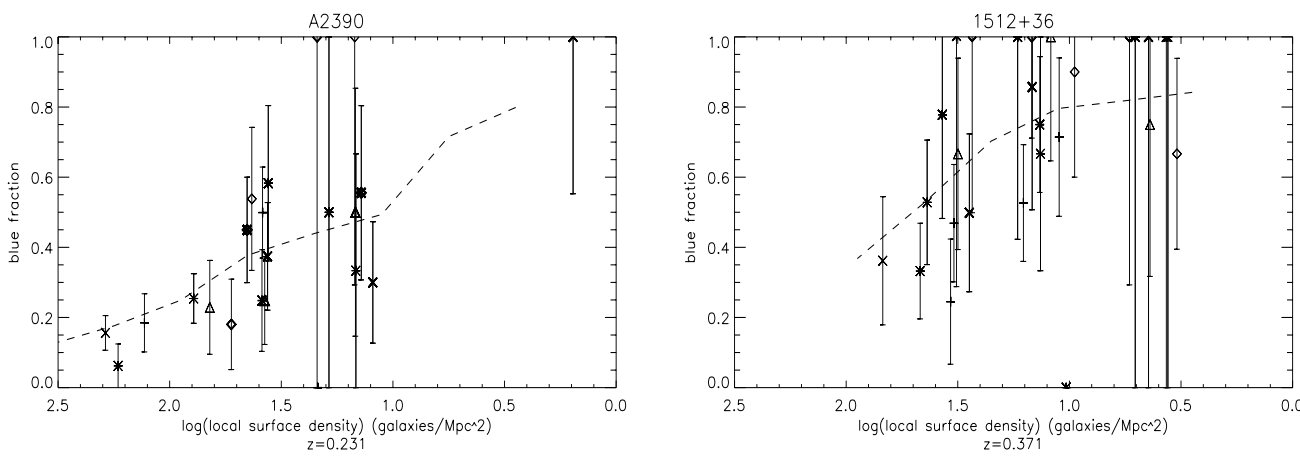

Figure 4. Blue galaxy fraction (dashed line) for the whole clusters as a function of local galaxy density for ( $a$ ) Abell 2390 (b) MS1512+36. Blue fractions in different groups are indicated by different symbols. The dependence of blue fraction on local galaxy density for groups is similar to that for the whole cluster. Clusters and groups at high redshift have larger blue fractions.

\section{References}

Connolly, A. J., Csabai, I., Szalay, A.S., Koo, D.C., Kron, R.G. \& Munn, J.A. 1995 AJ, 110-2655

Gómez et al. 2003 ApJ, 584-210

Kodama, T., Smail, I., Nakata, F., Okamura, S. \& Bower, R.G. 2001 ApJ, 562, L9

Yee, H.K.C., Ellingson, E. \& Carlberg, R.G. 1996 ApJS, 102-269

Yee, H.K.C., et al. 2000 ApJS, 475-492 